stamina numerosa (circiter 30 ), e tubo calycis vix exserta, inæqualia nec simplici serie inserta; filamentis rigidis, basi plus minusve in annulo bypogyno unitis; antheris ovatis bilobis, dorso affixis, introrsis.

Ovarium oblongum uniloculare, ovulo oblongo erecto subanatropo, chalaza laterali. Stylus rectus, staminibus longior. Stigma simplex, oblique expansum, papilloso-fimbriatum, latere decurrens.

Fructus, pericarpio membranaceo, oblongo-fusiformis, tubo calycis accreto et indurato, prismatico-pentagono, apice incrassato et constricto, inclusus. Tubus calycis, in fructu maturo, angulis visco tenacissimo indutis, lobis persistentibus dentiformibus coronatus.

Semen erectum, pericarpio conforme, liberum. Tegumentum membranaceum fuscum, pericarpio paulo crassius, uno latere, secundum longitudinem, subintroflexum et in lamina fusca (chalaza lineari), perispermio tenui molli mucoso involuta, extensum. - Embryo rectus, radicula inferiori, tigella cylindrica, cotyledonibus foliaceis subrotundis basi emarginato-cordatis, lateribus convolutis et perispermii vestigium involventibus.

\title{
Vieillakdia austro-Caledonica.
}

Arbor ramis crassis cylindricis, foliis quincuncie alternis, approximatis coriaceis, exstipulatis, petiolo angusto pollicari aut bipollicari, limbo subelliptico, integerrimo, penninervio, utrinque glabro ; floribus in corymbis terminalibus dispositis, calycibus coriaceis intus et extus puberulis.

Hab. in planitie insulæ Novæ-Caledoniæ, ubi clar. Vieillard primus invenit (herb. Mus. parisiensis et Mus. coloniarum).

Obs. - Les collections de la Nouvelle-Calédonie renferment encore des échantillons de deux arbres également voisins des Pisonia, du Vieillardia et du Calpidia ; mais ces échantillons, dépourvus de fruits, peuvent laisser des doutes sur leur détermination et nécessitent des comparaisons ubérieures avec les espèces de ce groupe propres à la Polynésie.

NOTE SUR LE GENRE CROSSOSTYLIS DE FORSTER, par MII. Ad. BRENGNIATET et Arthur Gisis.

Les collections formées par MM. Pancher et Vieillard à la Nouvelle-Calédonie comprennent, en bouton, en fleur et en fruit, une plante que nous avions reconnue pour appartenir au genre assez imparfaitement décrit par Forster sous le nom de Crossostylis. La grandeur de ses fleurs nous l'avait fait désigner sous le nom spécifique de grandiflora. M. Pancher, dans ses derniers envois, avait été conduit aussi à lui donner ce même nom.

Le Crossostylis biflora de Forster a été décrit et figuré récemment par M. Asa Gray dans la botanique du voyage du capitaine Wilkes (Unit. Stat. exploring expedition, p. 610, tab. 77); mais il ne l'a pas observé en fruit, 
et sa description de la fleur, comparée à des échantillons de cette même espèce, nous paraît inexacte en un point assez important de la structure de l'ovaire.

Le Crossostylis biflora et le Crossostylis grandiflora se ressemblent par tous les points essentiels de leur structure; ils diffèrent légèrement par la forme des sépales et des pétales, par le nombre des étamines qui est de 20 dans le $C$. biflora d'après Forster et $\mathbf{M}$. Asa Gray, que nous avons trouvé de 21 à 24 , suivant les fleurs, dans un échantillon de cette espèce de la NouvelleCalédonie, et qui est de 28 dans le $C$. grandiflora, une étant opposée devant chaque pétale et six devant chaque sépale. Les mêmes appendices se trouvent entre la base des étamines en dedans du tube du calice.

L'ovaire, qui est à moitié plongé dans le réceptacle, mais dont la surface supérieure convexe est marquée de stries rayonnantes, a été décrit et figuré par M. Asa Gray comme à 12 loges bi-ovulées; Forster indiquait le fruit comme uniloculaire. En examinant avec soin l'ovaire des deux espèces que nous avons eues à notre disposition, nous avons reconnu d'une manière bien positive, même sur des boutons jeunes, que les cloisons observées par le savant botaniste américain ne sont que des lames saillantes partant du fond de l'ovaire et de ses parois latérales, mais qui, à aucune époque, ne sont unies ni même rapprochées de la paroi supérieure libre de l'ovaire. Ce ne sont que des cloisons incomplètes, assez saillantes dans le $C$. biflora, ne formant que des nervures peu proéminentes dans le $C$. grandiflora. Ces lames indiquent cependant le nombre des carpelles constituants de l'ovaire, à chacun desquels correspondent deux ovules fixés à la colonne centrale qui traverse l'ovaire; cette colonne, très courte d'abord, s'allonge à mesure que le fruit se développe et que sa surface supérieure devient plus convexe. Les ovules, placés d'abord horizontalement, sont ensuite suspendus à l'extrémité de funicules roides assez allongés dans les graines fécondes. Le stigmate présente autant de petits lobes linéaires qu'il y a de carpelles, et ces lobes, diversenent groupés, sont assez souvent réunis en quatre faisceaux comme Forster l'avait observé. Les carpelles, dans le $C$. biflora, ne paraissent être qu'au nombre de 12 à 16 ; dans le $C$. grandiflora, ce nombre, est égal à celui des étamines, soit 28.

Le fruit, que Forster seul a fait connaître, nous paraît différer beauconp de sa description. Dans le $C$. grandiflora, il ressemble beaucoup par sa forme extérìeure à la figure de Forster, mais ce n'est nullement une baie indéhiscente. La partie supérieure convexe, formant comıne un couvercle hémisphérique marqué de stries rayonnantes, se détache par suite de la rupture de la colonne centrale au-dessus de l'insertion des graines; cette colonne centrale, qui persistait ainsi au milieu de la partie inférieure du fruit, portait, dans ceux que nous avons observés, beaucoup d'ovules avortés et un certain nombre de graines fertiles et mûres. Celles-ci étaient suspendues à l'extrémité de funicules roides et subulés qui se terminaient par une caroncule blanche spongieuse lobée qui recouvrait en partie la graine à testa noir et brillant; lorsqu'on détachait la graine, 
cette petite excroissance celluleuse ne l'accompagnait pas comme un arille, mais restait fixée au funicule. Leur présence dans des fruits dont beaucoup de graines seraient fertiles, formerait une sorte de pulpe qui a pu faire désigner ce fruit par Forster par les mots de bacea unilocularis polysperma. Quant à la graine elle-même, elle est ovale, à testa noir, lisse et brillant; elle renferme un périsperme charnu épais et un embryon droit placé dans l'axe de la graine. Sa radicule est dirigêe supérieurement vers le hile, et ses cotylédons, un peu plus larges que la tigelle, sont elliptiques, convexes et appliqués l'un contre l'autre.

Ces caractères de la graine confirment les rapports de ce genre avec les Légnotidées (1).

M. Chatin présente à la Sociètẻ un échantillon anomal d'Orchis maculata, récolté le 20 de ce mois dans la forêt de Rambouillet, près de Montfort-l'Amaury (Seine-et-0ise), et dont la tige, simple dans le bas, se bifurque sans fasciation préalable, pour porter deux épis cylindriques et semblables aux épis normaux. M. Chatin ajoute que l'on doit rapprocher ce fait d'une anomalie analogue observée par M. Duchartre sur une Tulipe (2).

(1) Depuis la lecture de cette note, nous avons observé, dans les collections de M. Vieillard $\left(\mathrm{n}^{\circ} \mathbf{4} 3\right)$, une troisième espèce de Crossostylis que nous désignerons par le nom de $C$. multiflora. Elle diffère des deux espèces précédentes : $1^{\circ}$ par des pédoncules plusieurs fois dichotomes, portant des fleurs beaucoup plus petites; $2^{\circ}$ par le nombre beaucoup moins considérable des parties de l'androcée et du pistil, qui sont les unes et les autres réduites à huit, 4 étamines étant opposées aux sépales et 4 aux pétales.

(2) Voyez le Bulletin, t. VII, p. 462. (Note ajoutée au moment de l'impression.)

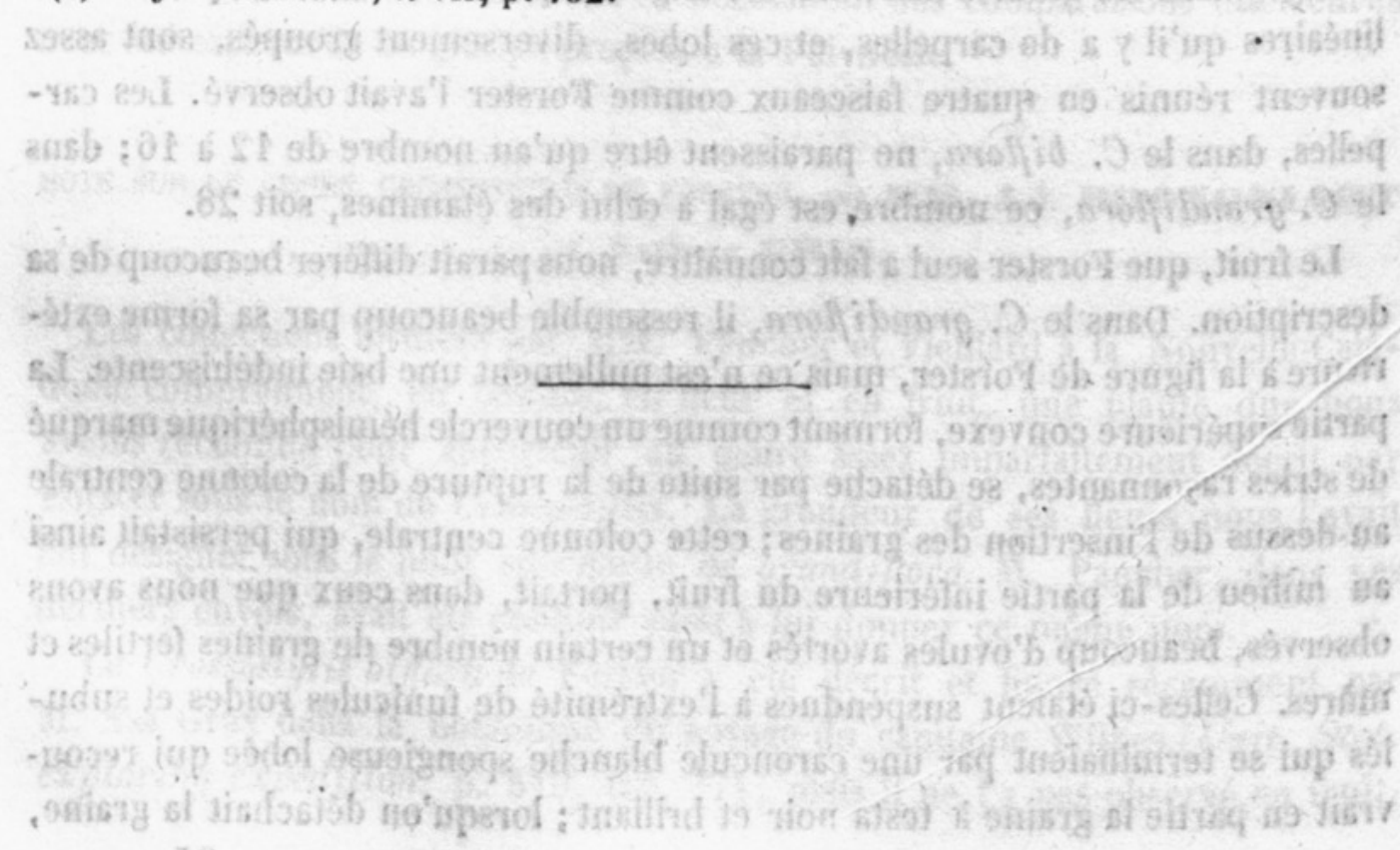




\section{$2 \mathrm{BHL}$ Biodiversity Heritage Library}

Brongniart, Adolphe and Gris, Arthur. 1861. "Note Sur Le Genre Crossostylis De Forster." Bulletin de la Société botanique de France 8, 376-378. https://doi.org/10.1080/00378941.1861.10829590.

View This Item Online: https://www.biodiversitylibrary.org/item/8632

DOI: https://doi.org/10.1080/00378941.1861.10829590

Permalink: https://www.biodiversitylibrary.org/partpdf/158347

\section{Holding Institution}

Missouri Botanical Garden, Peter H. Raven Library

\section{Sponsored by}

Missouri Botanical Garden

\section{Copyright \& Reuse}

Copyright Status: Public domain. The BHL considers that this work is no longer under copyright protection.

This document was created from content at the Biodiversity Heritage Library, the world's largest open access digital library for biodiversity literature and archives. Visit BHL at https://www.biodiversitylibrary.org. 